\title{
ESTUDO LONGITUDINAL DA FORCA EM IDOSAS PRATICANTES DE ATIVIDADE FISICA COM 70 ANOS E MAIS
}

\author{
Alexsander Vieira Guimarães ${ }^{1}$ \\ Marize Amorim Lopes² \\ Cíntia de la Rocha Freitas ${ }^{3}$ \\ Tânia Bertoldo Benedetti ${ }^{4}$
}

resumo

O objetivo do estudo foi verificar o desempenho da força dos membros superiores e inferiores em mulheres com idade igual e superior a 70 anos, praticantes regularmente de um programa de atividade física, por um período de quatro anos. Participaram 54 mulheres, com idade entre 70 a 85 anos (74,2 $\pm 3,3)$. Utilizou-se o teste de "resistência de força" (número de flexões de cotovelo) para avaliar a força dos membros superiores (FMS) e o teste "sentar e levantar" para avaliar a força dos membros inferiores (FMI), ambos da bateria "Sênior Fitness Tests - SFT". Os resultados da FMS e da FMI da primeira avaliação para os demais períodos destacam

1 Mestre em Educação Física. Doutorando na Universidade Federal de Santa Catarina (UFSC). E-mail: a.guimaraes51@yahoo.com.br

2 Doutora em Educação Física pela Universidade Federal de Santa Catarina (UFSC). E-mail: marize@cds.ufsc.br

3 Doutora em Educação Física pela Universidade Federal de Santa Catarina (UFSC). E-mail: cintiadelarocha@gmail.com

4 Doutora em Educação Física pela Universidade Federal de Santa Catarina (UFSC). E-mail: benedetti@cds.ufsc.br 
diferenças significantes $(p<0,05)$, de acordo com o teste de ANOVA com medidas repetidas. Portanto, participar regularmente de um programa de atividade física está contribuindo para que as mulheres desta faixa etária melhorem e/ou mantenham o desempenho da FMS e da FMl.

palavras-chave

Força Muscular. Atividade Física. Envelhecimento.

\section{Introdução}

O envelhecimento populacional mundial tem causado um profundo impacto nas sociedades do século XXI. Pois, associado ao envelhecimento humano, está com o aumento na prevalência das doenças crônicas e, consequentemente, dos gastos públicos. Neste sentido, torna-se necessário desenvolver ações com o intuito de propiciar boa condição de vida e saúde pelo maior tempo possível, especialmente de pessoas idosas, mantendo sua capacidade funcional (BENEDETTI et al., 2004; FERNADES et al., 2009).

Para manutenção da capacidade funcional, torna-se necessário prevenir a redução de capacidades físicas, como força muscular, capacidade aeróbia, coordenação e flexibilidade, relacionadas com o aumento da idade (CARDOSO; PRIOLI; BARELA, 2006; SINGH et al., 2006; CHODZKO-ZAJKO et al., 2009).

A força muscular é uma das capacidades físicas mais importantes e está associada à execução de diversas atividades cotidianas (MONTEIRO et al., 1999). Frontera et al. (2000), aponta declínio anual na força de membros inferiores em idosos entre 2,0 a 2,5\%. Harries e Bassey (1990) observaram redução de $15 \%$ por década na força muscular, especialmente entre a sexta e a sétima década de vida, com declínio mais acentuado em idades mais avançadas, de aproximadamente $30 \%$.

A prática de atividade física pode contribuir com a preservação e aumento da força e massa muscular, além de ter caráter preventivo, terapêutico e de promoção da saúde, devendo ser enfatizados exercícios resistidos e aeróbios de intensidade moderada (CHODZKO-ZAJKO et al., 2009). Os programas de atividades físicas oferecidas a idosos em diferentes locais, como universidades, prefeituras e centros de saúde, normalmente trabalham com ênfase a capacidade física da força (BORGES et al., 2008). 
Portanto, a avaliação da força em idosos pode assumir relativa importância para verificar se tais programas produzem melhoras sobre esta capacidade física e de quanto é o aumento, especialmente em idosos que participam há longo tempo em programas de atividades físicas. Desta forma, considera-se relevante oferecer dados a partir de um estudo longitudinal para que se elaborem estratégias, visando melhorias para a saúde e bem-estar dessas pessoas.

Diante do exposto, o objetivo do presente estudo foi verificar o desempenho da força dos membros superiores e inferiores em mulheres com idade igual e superior a 70 anos, praticantes de um programa regular de atividade física, por um período de quatro anos.

\section{Métodos}

Participaram deste estudo longitudinal, 54 mulheres com idade igual ou superior a 70 anos, que frequentaram regularmente o Programa de Atividade Física da Terceira Idade do Centro de Desportos (CDS) da Universidade Federal de Santa Catarina (UFSC), por um período regular de quatro anos. Os critérios de inclusão na amostra foram: mulheres com idade igual ou superior a 70 anos; ter participado de todas as avaliações; praticar regularmente do programa de atividades físicas supervisionadas, de intensidade moderada com duração de 50 minutos, frequência de duas vezes por semana e durante nove meses por ano, apresentando no mínimo $70 \%$ de assiduidade.

O Programa de Atividade Física da Terceira Idade do CDS-UFSC tem como principal objetivo oportunizar a prática saudável de atividades físicas e recreativas aos idosos, buscando a promoção da saúde, bem como ampliar o conhecimento nesta área, favorecendo a mudança de estilo de vida. As aulas eram realizadas no período letivo da universidade de março a dezembro, em duas sessões semanais, com duração de 50 minutos, desenvolvidas em três partes: 1) parte inicial: destinada ao aquecimento, com duração aproximada de $10 \mathrm{a}$ 15 minutos; 2) parte principal: eram trabalhadas as capacidades funcionais (resistência de força, flexibilidade, agilidade/equilíbrio dinâmico, resistência aeróbia e coordenação), com duração de 20 a 30 minutos, utilizando materiais como cordas, extensores, caneleiras, halteres, bolas, arcos (bambolês), steps e bastões; 3) parte final: focados os exercícios de respiração, alongamentos e relaxamento, com duração média de 10 a 15 minutos. 
Para avaliar o desempenho da força de membros inferiores e superiores, foram aplicados os seguintes testes, propostos pela bateria "Sênior Fitness Tests - SFT" (RIKLI; JONES, 1999):

Força de membros superiores (FMS): "flexão do cotovelo": O protocolo de execução do teste foi realizado da seguinte forma: a participante ficou sentada numa cadeira sem braços, com a mão dominante segurando o halter, pesando $1,8 \mathrm{~kg}$, e deveria realizar o maior número de flexões de cotovelo no tempo de 30 segundos, sendo concedidas duas tentativas com intervalo de descanso de um minuto, sendo registrado o melhor resultado.

Força de membros inferiores (FMI): "sentar e levantar": Verificado pelo teste de "sentar e levantar" da cadeira, estando com os braços cruzados sobre o peito, registrando o número total de execuções corretas num intervalo de 30 segundos, concedidas duas tentativas com intervalo de descanso de um minuto, sendo anotado o melhor resultado.

Os dados foram coletados por uma equipe devidamente treinada e composta por professores responsáveis pelo programa, que se fizeram presentes em todas as etapas do estudo e acadêmicos do curso de Educação Física da Universidade Federal de Santa Catarina, e realizados em cinco momentos, antes do início do programa (pré) e ao final de cada ano, ao longo de quatro anos (pós 1 , pós 2 , pós 3 , e pós 4 ).

Este estudo obteve aprovação do Comitê de Ética para seres Humanos da Universidade Federal de Santa Catarina, processo no $050 / 05$. Todas as idosas foram informadas sobre o objetivo da pesquisa e assinaram o termo de consentimento livre e esclarecido - TCLE.

\subsection{Análise estatística}

Foram utilizados os registros dos testes no Software CFI (Capacidade Funcional dos Idosos). Utilizou-se a estatística descritiva (média e desvio padrão) para caracterização dos valores de força muscular em cada momento. Para verificar o desempenho da força ao longo do tempo, foi utilizado o teste de ANOVA para medidas repetidas, com nível de significância de $5 \%(p<0,05)$. Os dados foram analisados com o auxílio do programa SPSS (versão 15.0). 
A média de idade das mulheres no início do programa foi de 74,2 \pm 3,3 (desvio-padrão) anos, com variação de 70 a 85 anos. Entre as idosas, 41 mulheres $(75,9 \%)$ eram viúvas, 46 (85,9\%) tinham ensino fundamental completo ou inferior e 32 (59,3\%), tinham renda familiar mensal entre dois e quatro salários mínimos. A massa corporal teve média de $64,0( \pm 10,5)$ quilogramas, a estatura de 153,6 $( \pm 5,1)$ centímetros e o índice de massa corporal de 27,1 $( \pm 3,9)$.

Na tabela 1, encontram-se os valores de média e desvio-padrão para a força de membros inferiores (FMI) e força de membros superiores (FMS) nos diferentes momentos analisados.

Tabela 1. Média e desvio-padrão ( \pm ) dos valores daforça de membros inferiores e de membros superiores (número de repetições nos testes), segundo os diferentes momentos de avaliação.

\begin{tabular}{|l|l|l|l|l|l|}
\hline Variáveis & Pré & Pós 1 & Pós 2 & Pós 3 & Pós 4 \\
\hline Idade (anos) & $74,2 \pm 3,3$ & $74,9 \pm 3,3$ & $75,9 \pm 3,3$ & $76,9 \pm 3,3$ & $77,9 \pm 3,3$ \\
\hline FMl (repetições) & $14,2 \pm 3,7$ & $16,1 \pm 3,5$ & $16,9 \pm 3,3$ & $18,2 \pm 3,8$ & $17,3 \pm 3,1$ \\
\hline FMS (repetições) & $15,2 \pm 3,6$ & $18,7 \pm 3,8$ & $19,9 \pm 3,7$ & $20,5 \pm 3,2$ & $20,3 \pm 3,4$ \\
\hline
\end{tabular}

FMl: força de membros inferiores; FMS: força de membros superiores.

Os valores percentuais na FMI e FMS, entre os diferentes momentos avaliados e o início da participação no programa, são apresentados na figura 1. Observou-se melhora na força muscular em todos os momentos em relação ao início do programa, tanto de membros inferiores, quanto de membros superiores. Na avaliação após o primeiro ano do programa (pós 1), já se observou aumento de $13,4 \%$ para FMI e de $23 \%$ para FMS. No último momento avaliado, após quatro anos, o aumento percentual da força foi de $21,8 \%$ e 33,6\% para FMI e FMS, respectivamente, quando comparados com o início do programa. 
Figura 1. Diferença percentual dos valores da força de membros inferiores (FMI) e força de membros superiores (FMS) nos diferentes momentos de avaliação em relação à avaliação inicial.

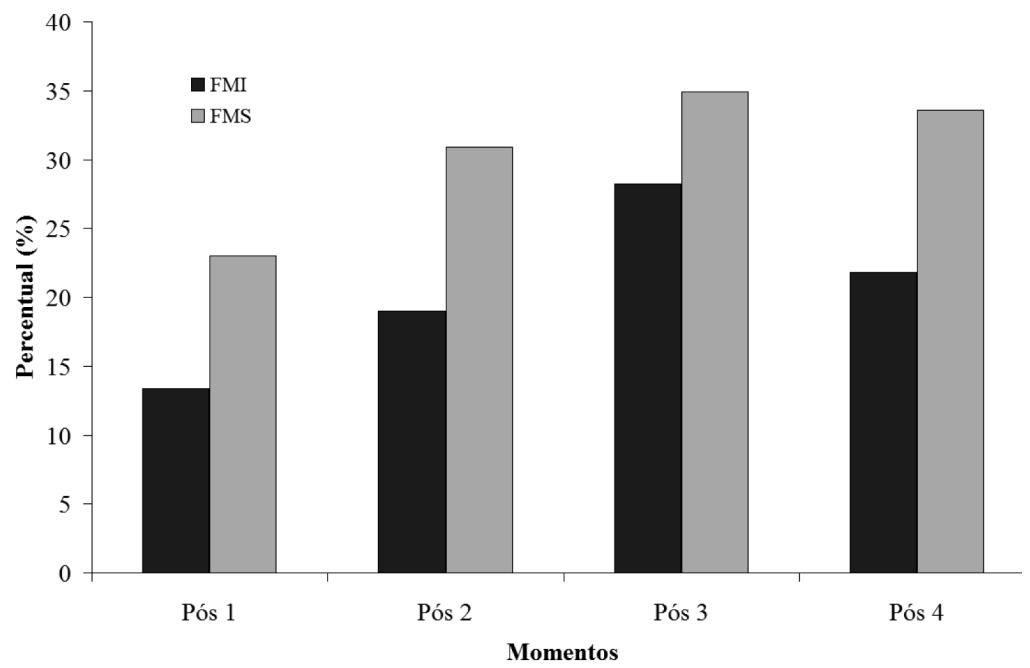

A figura 2 apresenta os resultados da FMS, de acordo com o teste de ANOVA com medidas repetidas, destacando aumento significante $(p<0,05)$ entre o início da participação no programa e os diferentes momentos avaliados. Os resultados também demonstram diferença estatística significante $(p<0,05)$ entre a segunda e a quarta avaliação.

Figura 2. Distribuição dos valores da força de membros superiores (FMS), segundo os diferentes momentos.

FMS

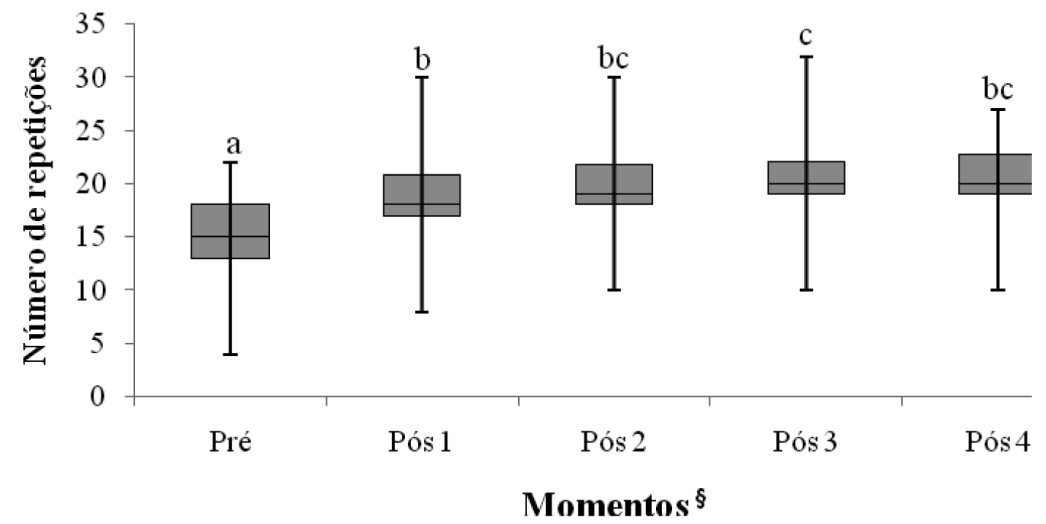


$\S$ Momentos com letras iguais não diferem significativamente ( $p>0,05)$.

Em relação à FMI, as diferenças entre os momentos estão apresentadas na figura 3. Observa-se diferença significante $(p<0,05)$, indicando aumento da força entre o início do programa (avaliação pré) em relação aos diferentes momentos (avaliações pós 1 , pós 2 , pós 3 e pós 4 ). Também houve diferença significante $(\mathrm{p}<0,05)$ no período pós 1 e pós 3 .

Figura 3. Distribuição dos valores da força de membros inferiores (FMI), segundo os diferentes momentos.

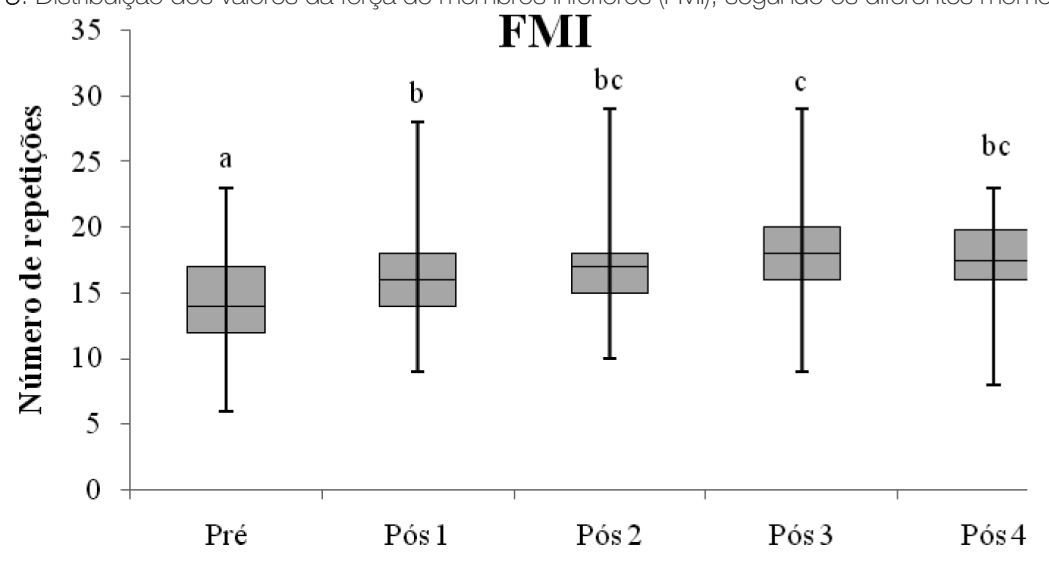

Momentos $\S$

$\S$ Momentos com letras iguais não diferem significativamente $(p>0,05)$. reation and Dance. Association Drive: Reston, 1990.

\section{Discussão}

Este estudo longitudinal teve como objetivo verificar o desempenho da força dos membros superiores e inferiores em mulheres com idade igual ou superior a 70 anos, participantes de um programa de atividade física supervisionada, por um período de quatro anos. Observou-se aumento na força tanto nos membros inferiores, quanto superiores, mesmo com o avançar da idade, em que normalmente ocorre uma acentuada redução de força muscular (HARRIES; BASSEY, 1990; FRONTERA et al., 2000).

No estudo realizado por Paulli et al. (2009), envolvendo 10 mulheres, com idade igual e superior a 60 anos, por 12 anos, também verificaram que a prática regular de atividade física pode manter ou mesmo melhorar a capacidade funcional, principalmente a força muscular. Da mesma forma, Matsudo 
et al. (2004), com um estudo longitudinal, de quatro anos, envolvendo 82 mulheres com idade entre 50 a 82 anos, encontraram melhora e/ou manutenção da FMS, durante o período de quatro anos, mostrando resultados similares ao presente estudo.

Os resultados deste estudo evidenciam ganhos significativos na FMI entre as avaliações. Paulli et al. (2009) mostraram melhora na força muscular em mulheres idosas, durante a participação no programa de atividade física, e declínio das capacidades relacionadas à extremidade inferior no grupo controle, que não eram praticantes do programa de atividade física. Resultados como esses, assim como os do presente estudo, ressaltam a relevância da prática de atividade física regular e orientada para evitar as perdas referentes à força muscular, principalmente de membros inferiores.

O estudo de Raso, Matsudo e Matsudo (2001), realizado com programa de exercícios com pesos livres durante 12 semanas, três vezes por semana, demonstrou aumento de pelo menos $40 \%$ na força muscular em mulheres idosas. Frontera et al. (2000) apontam que programas de exercícios físicos de força podem contribuir para ganhos entre 15 a $175 \%$.

Como a força muscular está associada a diversas atividades diárias, como levantar-se da cadeira, habilidade de subir degraus e velocidade da marcha, sua redução pode comprometer a independência dos indivíduos (FRONTERA et al., 2000; DESCHENES, 2004; PEIFFER et al, 2010). Essa redução ocorre indiferente do sexo, entretanto, nos diversos estágios da vida as mulheres apresentam menor força muscular que os homens (DESCHENES, 2004; JANSSEN et al., 2000), bem como nos músculos dos membros superiores e inferiores.

Com o avanço da idade, parece ocorrer decréscimo maior da força nos membros inferiores (JANSSEN et al., 2000; LYNCH et al., 1999; PAULLI et al., 2009). Uma possível explicação é que enquanto os membros superiores são inervados apenas pelo plexo (conjunto de nervos) braquial, os membros inferiores são inervados pelo plexo lombar e sacral, envolvendo maior quantidade de músculos. Isso pode gerar maior dificuldade de contração entre os músculos, encurtamento muscular, e contribuir no aumento da fraqueza muscular (KEND A L L et al., 2007). Outro fato que pode contribuir é o desempenho funcional em tarefas que envolvam esforços musculares dos membros inferiores, que podem declinar mais do que os membros superiores em idosos de ambos os sexos, mesmo nas pessoas praticantes de exercícios físicos regularmente (ONDER et al.,2002).

Alterações neuromusculares relacionadas com a idade estão relacionadas com o decréscimo na força muscular, ocasionada principalmente pela 
redução de motoneurônios alfa e do número de unidades motoras das fibras musculares (ONDER et al., 2005). A massa muscular pode diminuir aproximadamente seis por cento por década, sendo que aos 80 anos a pessoa pode ter perdido entre 30 a 40\% de sua massa corporal total (FRONTERA et al., 2000).

Deste modo, a avaliação da força muscular durante o envelhecimento, pode ser considerada um dos parâmetros mais importantes para analisar a capacidade funcional. No estudo longitudinal de três anos realizado por Onder et al. (2005), os autores analisaram 884 mulheres, e constataram que as medidas de força dos membros superiores e inferiores foram significativas para a predição de delimitações funcionais, e afirmam que a sarcopenia associada ao envelhecimento é inevitável, e acontece de forma lenta e progressiva na idade superior a 65 anos. Guralnik, Ferrucci e Pieper (2000) ao avaliarem 4.588 idosos de ambos os sexos, utilizando o teste de "sentar e levantar" da bateria de testes SFT, observaram que o desempenho dos membros inferiores prevê precisamente a incapacidade em populações de idosos. Ostchega et al. (2000) pesquisaram 5.403 idosos de ambos os sexos, com o mesmo teste de FMI, "sentar e levantar" da cadeira, ressaltando que pode ser uma medida confiável para avaliar a FMI em idosos.

É importante salientar que incentivar a prática de atividade física regular para os idosos em idade avançada é fundamental para sua autonomia e controle de suas capacidades funcionais. Entretanto, este estudo apresenta algumas limitações, principalmente em relação à falta de controle/ periodização das aulas durante o Programa de Atividade Física da Terceira Idade do CDS - UFSC. Além disso, não houve grupo controle para comparação dos resultados. Entretanto, os resultados obtidos indicam que, mesmo sem estes controles, foram observadas melhoras significantes e/ou manutenção na força de membros superiores e inferiores das participantes.

\section{Conclusão}

Este estudo evidenciou que o desempenho da força muscular de membros superiores e inferiores, em mulheres idosas, pode ser beneficiado com a prática regular de atividade física, considerando que o ato de exercitar-se contribui para o desempenho do sistema musculoesquelético, essencial para a independência e autonomia das pessoas idosas. Deste modo, um programa regular e orientado de atividade física contribui para que as idosas, 70 anos ou mais de idade, mantenham e/ou melhorem o desempenho da força, mesmo em idade avançada. 
LONGITUDINAL STUDY OF STRENGTH IN WOMEN WHO PRACTICE PHYSICAL ACTIVITY OVER 70 YEARS OLD

\section{abstract}

The objective of this study was to verify the strength performance in women 70 years old or older who practice physical activity. To access the upper members strength (UMS), it was used the test "strength endurance" and the lower members strength (LMS) was accessed using the "sit down and stand up" test, both from the Senior Fitness Tests - SFT battery. The study included 54 women 70 to 85 years old $(74,2 \pm 3,3)$. The UMS and LMS results, when comparing the first evaluation with the following periods, showed significant differences $(0<0,05)$, according to the ANOVA measures. We conclude, therefore, that participating in a regular physical activity program contributes to the improvement and maintenance of performance on the FMS and FMI in women 70 years old and older.

keywords

Muscular Strength. Physical Activity. Aging.

referências

BENEDEITI, Tânia Rosane Bertoldo et al. Valores normativos de aptidão funcional em mulheres de 70 a 79 Anos. Revista Brasileira de Cineantropometria e Desempenho Humano, Florianópolis, v. 9, n. 1, p. 28-36, 2004.

BORGES, Lucélia Justino; CARDOSO, Adilson Santana; BENEDEITI; Tânia Rosendo Bertoldo et al. Teste de resistência de força de membros superiores para idosos: comparação entre halteres com pesos diferentes. Revista Brasileira de Cineantropometria e Desempenho Humano, Florianópolis, v. 10, n. 3, p. 261-265, 2008.

CARDOSO, Aline Steckelberg; PRIOLI, Ana Caroline; BARELA, José Ângelo. Atividade física e acoplamento entre percepção e ação no controle postural de idosos. Motricidade, Vila Real, v. 2, n. 3, p. 178-191, 2006.

CHODZKO-ZAJKO, Wojtek; PROCTOR, David; FIATARONE, Maria Singh et al. American College of Sports Medicine position stand. Exercise and physical activity for older adults. Medicine and Science in Sports and Exercise, Indianapolis, v. 41, n. 7, p. 15101530, July. 2009.

DESCHENES, Michael. Effects of aging on muscle fibre type and size. Sports Medicine, v. 34, n. 12 , p. $809-824,2004$

FERNADES, Helder Miguel et al. A Influência da actividade física na saúde mental positiva de idosos. Motricidade, Vila Real, v. 5, n. 1, p. 33-50, 2009. 
FRONTERA, Walter R.; HUGHES, Virginia; FIELDING, Roger et al. Aging of skeletal muscle: a 12-yr longitudinal study. Journal of Applied Physiology, Bethesda, v. 88, n. 4, p. $1321-1326,2000$.

GURALNIK, Jack; FERRUCCI, Luigi; PIEPER, Carl. Lower extremity function and subsequent disability: consistency across studies, predictive models, and value of gait speed alone compared with the short physical performance battery. Journal of Gerontology Series A Biological Sciences Medical Sciences, Washington, v. 55, p. 4, p. 221-231, 2000.

HARRIES, Ursula; BASSEY, Eileen Joan. Torque-velocity relationships for the knee extensors in women in their 3rd and 7th decades. European Journal of Applied Physiology and Occupational Physiology, Berlin, v. 60, n. 3, p. 187-190, 1990.

JANSSEN, lan; HEYMSFIELD, Steven; WANG, Zimian et al. Skeletal muscle mass and distribuition in 468 men and women aged 18-88 yr. Journal of Apllied Physiology, Bethesda, v. 89, n. 1, p. 81-88, 2000.

KENDALL, Florence Petersen; MCCREARY, Elizabeth Kendall; PROVANCE, Patricia Geise et al. Músculos: provas e funções com postura e dor. Barueri - SP: Manole, 2007.

LYNCH, Nicole; MEITER, Jeffrey; LINDLE, Rosemery et al. Muscle quality. I. Age-associated differences between arm and leg muscle groups. Joumal of Apllied Physiology. Bethesda, v. 86, n. 1, p. 188-194. 1999.

MATSUDO, Sandra Marcela; MARIN, Rosângela; FERREIRA, Marcela et al. Estudo longitudinal-tracking de 4 anos - da aptidão física de mulheres da maioridade fisicamente ativas. Revista Brasileira de Ciência e Movimento, Taguatinga, v. 12, n. 3, p. 47-52, setembro. 2004.

MONTEIRO, Walace David; AMORIM, Paulo Roberto; FARJALLA, Renato et al. Força muscular e características morfológicas de mulheres idosas praticantes de um programa de atividades físicas. Revista Brasileira de Atividade Física e Saúde, Pelotas, v. 4, n. 3, p. 20-28, 1999.

ONDER, Graciano; PENNINX, Brenda; FERRUCCI Luigi et al. Measures of physical performance and risk for progressive and catastrophic disability: Results from the Women's Health and Ageing Study. The Joumal of Gerontology Series A Biological Sciences Medical Sciences, Washington, v. 60, n. 1, p. 74-79, 2005.

ONDER, Graciano; PENNINX, Brenda; LAPUERTA, Pablo et al. Change in Physical Performance Over Time in Older Women: The Women's Health and Aging Study. The Joumal of Gerontology Series A Biological Sciences Medical Sciences, Washington, v. 57 , n. 5, p. 289-293, 2002.

OSNESS, Wayne; ADRIAN, Marlene; CLARK, Bruce et al. Functional Fitness Assessment for Adults Over 60 Years (A Field Based Assessment). The American Alliance for Health, Physical Education, Rec

OSTCHEGA, Yechiam; HARRIS, Tamara; HIRSCH, Rosemery et al. The prevalence of functional limitations and disability in older persons in the US: data from the National Health and Nutrition Examination Survey III. Journal of the American Geriatrics Society, New York, v. 48, n. 9, p. 1132-1135, 2000.

PAULLI, José Rodrigo; SOUZA, Luciana Santos; ZAGO, Anderson Saranz et al. Influência de 12 anos de Prática de Atividade Física Regular em Programa Supervisionado para Idosos. Revista Brasileira de Cineantropometria e Desempenho Humano, Florianópolis, v. 11, n. 3, p. 255-260, 2009.

PEIFFER, Jeremiah; GALVÃO, Daniel; GIBBS, Zoe et al. Strength and functional characteristics of men and women 65 years and older. Rejuvenation Research, New Rochelle v. 13, n. 1, p. 75-82, Feb. 2010. 
Recebido: 24/07/2012

1ª Revisão: 01/10/2012

$2^{a}$ Revisão: 24/11/2012

Aceite Final: 02/12/2012
RASO, Vagner, MATSUDO Sandra Marcela; MATSUDO, Victor. A força muscular de mulheres idosas decresce principalmente após oito semanas de interrupção de um programa de exercícios com pesos livres. Revista Brasileira de Medicina do Esporte, São Paulo, v. 7, n. 6, p. 177-186, nov/dez. 2001

RIIKLI, Roberta; JONES, Jessie. Development and validation of functional fitness test for community-residing older adults. Joumal of Aging and Physical Activity, Champaign, v. 7 , n. 2, p. 129-181, 1999

SINGH, Amika S et al. Cross-sectional relationship between physical fitness components and functional performance in older persons living in long-term care facilities. BMC Geriatrics, London, v. 6, n. 4, p. 1-9, 2006. 